Data sources Cochrane Hepato-Biliary Group controlled trials register, Cochrane Controlled Trials Register, MEDLINE, LILACS, SCI Expanded, Embase and http://clinicaltrials.gov/ until January 2010; reference lists of relevant articles and authors were searched. Review methods The methodological approach outlined in the Cochrane Handbook was followed. Randomised trials comparing antibiotics with placebo, no intervention, non-absorbable disaccharides, another antibiotic or any other active treatment for HE were included. The primary outcome measures were no improvement of HE and all-cause mortality.

Results Twenty-eight studies were included in this review; most had a small sample size and were methodologically of a low quality. Two studies $(\mathrm{N}=132)$ compared antibiotics with placebo or no intervention, reporting no differences in primary outcomes between groups. Twelve studies $(\mathrm{N}=708)$ compared antibiotics with nonabsorbable disaccharides, reporting no difference in all-cause mortality ( $R R=1.11$ (95\% CI 0.60 to 2.07$)$ ) but a marginally significant reduction in the risk of "no improvement" (RR 0.83 (0.69 to 1.00$)$ ). In sensitivity analysis, this finding was significant in trials evaluating chronic encephalopathy ( $R R 0.50$ (0.28 to 0.89)) and in trials with co-administration of cathartic agents (RR 0.82 (0.67 to $0.99)$ ). Ten trials $(\mathrm{N}=336)$, compared different antibiotic regimens, most evaluating rifaximin and neomycin. Rifaximin reduced the risk of no improvement compared to neomycin, but not significantly so (RR 0.61 (0.34 to 1.09$)$ ).

Conclusions Insufficient evidence exists to support or refute the use of antibiotics for HE. Antibiotics were slightly superior to nonabsorbable disaccharides at improving $\mathrm{HE}$, with borderline significance and possible effect modification.

\section{P2-77 BEHAVIOUR PROBLEMS AND PREVALENCE OF ASTHMA SYMPTOMS AMONG BRAZILIAN CHILDREN}

doi:10.1136/jech.2011.142976i.12

\begin{abstract}
${ }^{1,2} \mathrm{C}$ Feitosa, ${ }^{*} \mathrm{D}$ Santos, ${ }^{3} \mathrm{M}$ B do Carmo, ${ }^{1} \mathrm{~L}$ Santos, ${ }^{4} \mathrm{C}$ Teles, ${ }^{2} \mathrm{~L}$ Rodrigues, ${ }^{1}$ M Barreto. 'Institute of Collective Health, Federal University of Bahia, Salvador, Bahia, Brazil; ' London School of Hygiene and Tropical Medicine, LSHTM, London, UK; ${ }^{3}$ Institute of Psychology, University of São Paulo, São Paulo, Brazil; ${ }^{4}$ Institute of Statistics, State University of Feira de Santana, Feira de Santana, Bahia, Brazil
\end{abstract}

Introduction Asthma is the most common chronic disease in childhood and has been designated a public health problem due to the increase in its prevalence in recent decades, the amount of health service expenditure it absorbs and an absence of consensus about its aetiology. The relationships among psychosocial factors and the occurrence, symptomatology, and severity of asthma have recently been considered. There is still controversy about the association between asthma and child's mental health, since the pathways through which this relationship is established are complex and not well researched. This study aims to investigate whether behaviour problems are associated with the prevalence of asthma symptoms in a large urban centre in Latin America.

Methods Cross-section study of 869 children between 6 and 12 years old, residents of Salvador, Brazil. The International Study of Allergy and Asthma in Childhood (ISAAC) questionnaire was used to evaluate prevalence of asthma symptoms. The Child Behaviour Checklist (CBCL) was employed to evaluate behavioural problems. Results $19.26 \% \quad(n=212)$ of the children presented symptoms of asthma. We found good evidence of an association between behaviour problems and asthma symptoms (PR: 1.53 ; 95\% CI 1.13 to 2.08 . $\mathrm{p}=0.007$ ) and this association remained statistically significant after adjustment for sex, age, maternal education, income, parental asthma, minor psychiatric disorders in the mother, Alcohol use, allergens in dust and smoking (PR: 1.43 ; 95\% CI 1.10 to 1.85. $\mathrm{p}=0.01$ ). Conclusion These results suggest an association between behavioural problems and paediatric asthma, and support the inclusion of mental healthcare in the provision of services for asthma morbidity.

\section{P2-78 QUALITY OF LIFE IN BRAZILIAN WOMEN WITH BREAST CANCER: ASSOCIATION WITH THE SOCIAL ENVIRONMENT}

doi:10.1136/jech.2011.142976i.13

${ }^{1}$ D B Ferreira, ${ }^{* 1} \mathrm{R}$ Koifman, ${ }^{2} \mathrm{~A}$ Bergmann. ${ }^{1}$ National School of Public Health, Oswaldo Cruz Foundation, Rio de Janeiro, Brazil; ${ }^{2}$ National Cancer Institute, Rio de Janeiro, Brazil

Introduction The incidence of breast cancer is increasing in Brazil. Breast cancer is a major cause of morbidity and mortality with major social and epidemiological impact. The aim of this study is to evaluate the association between social support before treatment for breast cancer and quality of life (QOL) 6 months after starting treatment.

Methods Eligible participants were women who were treated for incident breast cancer in the National Cancer Institute between June 2009 and March 2010. EORTC OLQ-C30 was used to measure QOL. MOS-SSS was used to assess social support prior to treatment. Bivariate analyses of dichotomous variables were carried out and ORs with 95\% CI were presented.

Results 195 women were evaluated. Good emotional support was a protective factor for overall health (OR 0.42 (95\% CI 0.23 to 0.79 )) and scale functionality (OR 0.36 (0.18 to 0.71$)$ ). Information support was associated with better global health (OR 0.41 ( 0.22 to $0.76)$ ), functionality (OR 0.35 (0.18 to 0.70$)$ ) and symptoms scale (OR 0.38 (0.18 to 0.77$)$ ). Women who reported good emotional support were $70 \%$ less likely to report general health problems 6 months after starting treatment $(0.13-0.65)$.

Conclusion The results of this study suggest that a good social environment prior to treatment is positively associated with $\mathrm{QOL}$ 6 months after starting treatment in women with breast cancer, underscoring the importance of assessing social environment.

\section{P2-79 THE IMPACT OF SOCIAL ENVIRONMENT ON QUALITY OF LIFE IN ELDERLY WOMEN WITH BREAST CANCER IN BRAZIL}

doi:10.1136/jech.2011.142976i.14

${ }^{1} \mathrm{D}$ B Ferreira, ${ }^{*} \mathrm{R}$ Koifman, ${ }^{2} \mathrm{~A}$ Bergmann. ${ }^{1}$ National School of Public Health, Oswaldo Cruz Foundation, Rio de Janeiro, Brazil; ${ }^{2}$ National Cancer Institute, Brazil, Rio de Janeiro, Brazil

Introduction Studies suggest that social support is associated with improved health outcomes in cancer patients. The relationship between social environmental and health outcomes in elderly Brazilian women with breast cancer has not been investigated. This study aimed to explore the association between social support prior to treatment in a reference centre in Brazil and quality of life (QOL) in elderly women and women under 60 years of age with breast cancer.

Methods This study included women with breast cancer who started oncology treatment at National Cancer Institute between June 2009 and March 2010. The Medical Outcomes Study-Social Support Survey was used to assess social support prior to treatment. EORTC QLQ-C30 and BR23 module were used to measure QOL. Bivariate analyses of dichotomous variables and independent variables were carried out and RRs with CIs of $95 \%$ presented.

Results 195 women were examined of whom 46.2\% (90) were elderly. Older women who reported good emotional support had lower risk of presenting the worst score of global health (RR 0.69 (95\% CI 0.55 to 0.88$)$ ), functionality (RR 0.59 (0.38 to 0.91$)$ ) and 
symptoms (RR 0.25 (0.10 to 0.60$)$ ) of C30 questionnaire. Emotional support was negatively associated with global health in women aged under 60 years (RR 0.65 (0.50 to 0.85$)$ ).

Conclusions In this population social environment is positively associated with QOL; this effect is greatest in elderly women.

\section{P2-80 EARLY COMPLICATIONS OF BREAST CANCER TREATMENT AND OUALITY OF LIFE IN BRAZILIAN WOMEN}

doi:10.1136/jech.2011.142976i.15

${ }^{1} \mathrm{D}$ B Ferreira, ${ }^{* 1} \mathrm{R}$ Koifman, ${ }^{2} \mathrm{~A}$ Bergmann. ${ }^{1}$ National School of Public Health, Oswaldo Cruz Foundation, Rio de Janeiro, Brazil; ${ }^{2}$ National Cancer Institute, Rio de Janeiro, Brazil

Introduction Post-operative complications are a major cause of morbidity in women with breast cancer. The aim of this study was to evaluate the association between quality of life (QOL) before treatment for breast cancer and early post-operative complications in the National Cancer Institute-Brazil.

Methods Participants were women with unilateral breast cancer, requiring oncological treatment in National Cancer Institute-Brazil between June 2009 and March 2010. QOL before treatment was assessed using EORTC QLQ-C30 and BR23 module. Information about early post-operative complications (axillary web syndrome, pain and subjective symptoms related to early oedema in the upper limbs) were obtained by physical examination 30 days after surgery, collected using a standardised form. Bivariate analyses of dichotomous variables and independent variables were carried out and ORs, with $95 \%$ CIs present.

Results 181 women were studied. Women who reported good health overall were $57 \%$ less likely to experience early subjective oedema ( $95 \%$ CI 0.20 to 0.96$)$. Symptoms scale of BR23 was negatively associated with pain (OR=0.11; 95\% CI 0.21 to 0.73$)$. Patients with good functionality-C30 were less likely to experience pain (OR 0.66 $95 \%$ CI (0.15 to 0.75$)$ ). There was no statistically significant association with axillary web syndrome.

Conclusions In this study pre-treatment QOL in women with breast cancer was a useful predictor of early post-operative complications.

\section{P2-81 THE IMPACT OF SOCIAL ENVIRONMENT ON EARLY COMPLICATIONS IN WOMEN WITH BREAST CANCER IN A REFERENCE CENTRE IN BRAZIL}

doi:10.1136/jech.2011.142976i.16

${ }^{1} \mathrm{D}$ B Ferreira, ${ }^{* 1}$ R Koifman, ${ }^{2}$ A Bergmann. ${ }^{1}$ National School of Public Health, Oswaldo Cruz Foundation, Rio de Janeiro, Brazil; ${ }^{2}$ National Cancer Institute, Rio de Janeiro, Brazil

Introduction Studies suggest that good social support is associated with a reduction in post-treatment complications in breast cancer. The aim of this study is to evaluate the association between pretreatment social support and early complications after surgery (pain and subjective symptoms related to early oedema in the upper limbs) in women with breast cancer.

Methods Participants were women with breast cancer and that presented to the Brazilian National Cancer Institute between June 2009 and March 2010. MOS-SSS was used to assessment of social support before oncology treatment. Information about early postoperative complications were obtained by physical examination 30 days after surgery and collected in a standardised form. Bivariate analysis between dichotomous outcome and selected independent variables were conducted and RRs with $95 \%$ CIs were presented.

Results 181 eligible women were studied. Patients with good emotional and affective support had a $76 \%$ (95\% CI 0.10 to 0.57 ) reduced risk and $62 \%$ (0.12 to 0.64$)$ reduced risk of pain respectively. Better material support was associated with a $73 \%$ (0.11 to 0.623 ) reduction in the risk of pain. No significant association with early oedema was observed.

Conclusions Good social support was associated with reduced risk of post-operative complications in women undergoing treatment for breast cancer.

\section{P2-82 PREGNANCY, EXPOSURE TO PESTICIDES AND INFANT LEUKAEMIA IN BRAZIL}

doi:10.1136/jech.2011.142976i.17

${ }^{1} \mathrm{~J} D$ Ferreira, ${ }^{*} \mathrm{~A}$ C Couto, ${ }^{2} \mathrm{M}$ do Socorro Pombo-de-Oliveira, ${ }^{1} \mathrm{~S}$ Koifman. ${ }^{1}$ National School of Public Health, Oswald Cruz Foundation - Fiocruz, Rio de Janeiro, Brazil, ${ }^{2}$ National Institute of Cancer - Inca, Rio de Janeiro, Brazil

Infant Leukaemia is a rare haematological neoplasm associated with MLL gene rearrangements. Maternal exposure during pregnancy to pesticides, hormones, dipyrone, and topoisomerase-II DNA inhibitors and birth weight were associated risk factors. This is a hospitalbased multicenter case-control study, and were interviewed mothers of 252 IL cases and of 423 controls. Data were obtained regarding environmental maternal exposure during periconceptional, pregnancy and breast-feeding periods, including exposure to pesticides. Unconditional logistic regression was performed and ORs on the association between maternal pesticides exposure and IL, including their $95 \%$ CIs, were ascertained after adjustment to hormonal intake during pregnancy, mother's age, mother's level of education, birth weight and infant's skin colour. An adjusted OR, 2.39, 95\% CI 1.63 to 3.51 was observed for the association between IL and maternal exposure to pesticides during pregnancy, being higher for acute myeloid leukaemia (adjusted OR 3.50, 95\% CI 2.01 to 6.11). The use of pyrethroids during pregnancy revealed an OR 2.18 (95\% CI 1.44 to 3.29), while the use of other pesticides showed an OR $3.61(95 \%$ CI 1.69 to 7.73 ). The reported household use of pesticides revealed an adjusted OR 2.25 (95\% CI 1.48 to 3.43 ), and agriculture exposure showed an adjusted OR 9.26, (95\% CI 2.82 to 30.4). Mixed exposure to different pesticides showed an adjusted OR, 3.83 (95\% CI 1.33 to 11.0) and the exposure to different chemical classes revealed an adjusted OR, 8.87 (95\% CI 1.55 to 50.6). The observed results seem to support the hypothesis that mothers' household use of pesticides and other contaminants may be involved in the aetiology of infant leukaemia.

\section{P2-83 ENVIRONMENTAL EXPOSURES AND CHILDHOOD LEUKAEMIA: AN EXPLORATORY ANALYSIS IN BRAZIL}

doi:10.1136/jech.2011.142976i.18

${ }^{1} \mathrm{~J} D$ Ferreira, ${ }^{*} \mathrm{~A}$ C Couto, ${ }^{2} \mathrm{M}$ do Socorro Pombo-de-Oliveira, ${ }^{1} \mathrm{~S}$ Koifman. ${ }^{1}$ National School of Public Health, Oswald Cruz Foundation - Fiocruz, Rio de Janeiro, Brazil, ${ }^{2}$ National Institute of Cancer - Inca, Rio de Janeiro, Brazil

The aim of this study was to explore the unapparent relationships that several factors related to environmental exposures and individual characteristics existing in our environment seem to be involved with childhood leukaemia. From a database of clinical and epidemiological data obtained from hospital-based multicenter casecontrol study on risk factors for childhood leukaemia, exploratory multivariate analysis was performed the principal component and factor analysis. Further, an unconditional logistic regression was carried out aiming to ascertain the magnitude of association between the selected factors, and their composing variables, with childhood leukaemia. The model displaying the highest power explained $52 \%$ of the total variance, including three factors, each one 